\title{
Measuring Operator's Pain: Toward Evaluating Musculoskeletal Disorder at Work
}

\author{
Eya Barkallah \\ University of Quebec at \\ Chicoutimi \\ Chicoutimi, Canada \\ Eya_Barkallah1@uqac.ca
}

\author{
Martin J.-D. Otis \\ University of Quebec at \\ Chicoutimi \\ Chicoutimi, Canada \\ Martin_Otis@uqac.ca
}

\author{
Suzy Ngomo \\ University of Quebec at \\ Chicoutimi \\ Chicoutimi, Canada \\ Suzy_Ngomo@uqac.ca
}

\author{
Michel Heraud \\ University of Quebec at \\ Chicoutimi \\ Chicoutimi, Canada \\ Michel_Heraud@uqac.ca
}

\begin{abstract}
Musculoskeletal disorders (MSDs) have affected an increasing number of people in the active general population. In this perspective, we developed a measuring tool taking muscle activities in certain regions of the body, standing posture taking the center of pressure under the feet and feet positions. This tool also comprises an instrumented helmet containing an electroencephalogram (EEG) to measure brain activity, and an accelerometer reporting the movements of the head. Then, our tool comprises both non-invasive instrumented insole and safety helmet. Moreover, the same tool measures muscular activities in specific regions of the body using an electromyogram (EMG). The aim is to combine all the data in order to identify consistent patterns between brain activity, postures, movements and muscle activity, and then, understand their connection to the development of MSDs. This paper presents three situations reported to be a risk for MSDs and an analysis of the signals is presented in order to differentiate adequate or abnormal posture.
\end{abstract}

Keywords- Musculoskeletal disoders; posutre recognition; IMU; Center of Pressure.

\section{INTRODUCTION}

A great number of workers within industrialized countries are affected, among which $27,1 \%$ in Norway, $33,7 \%$ in the United Kingdom and 47,8\% in Quebec [1]. New ways to help the comprehension of the underlying physiopathological mechanisms in the persistence of MSDs are emerging. It has therefore been shown that some lesions caused by MSDs might be associated with, among other symptoms, a cerebral physiological signature specific to the lesion [2-4]. Musculoskeletal disorders (MSDs) in the neck-shoulder area have accounted an increasing number of people in the active general population. In fact, this number annually grows at a rate going from $10 \%$ to $50 \%$ [1]. Every year, between $11 \%$ and $14,1 \%$ of employees do not go to work because of neck pain [1, 5]. This project suggests designing a new tool for improving detection and prevention of MSD in real situation.

Besides, a lot of studies have clearly shown a significant association between working in a standing position and the presence of MSDs [6, 7]. Indeed, articles written about working in this condition, show that the over-weight endured by the anatomical structures supporting the weight of the body could in some way explain the outbreak of MSDs such as low back pain $[8,9]$.
It is clear that the first anatomical structures which support the weight of the body in a standing position are the feet. Surprisingly, posture adjustments of feet have never been taken into account in analysis in a real context of work. Accordingly, a literary review have indicated that the majority of interventions at work remain insufficient in order to eliminate MSDs [10], which is alarming. This could, however, be linked to the fact that very few data exist in order to better understand the physiopathological mechanisms which might explain the chronical aspect of these affections. In our work, we precisely tempted to include measurements like head movements, muscles activity (especially in the neck/shoulder area), feet positions (center of pressure) and movements, as well as the worker's posture to better understand those mechanisms.

Furthermore, recent studies have shown that MSDs are not merely local affections at the affected area, but that these lesions are coupled with a cerebral physiological signature specific to the lesion [2-4]. It has also been proven that different tasks require different levels of cerebral alert for an optimal performance [11]. In fact, according to the empirical law of Yerkes-Dodson, while routine tasks are made easier by a high alert level, complex tasks requiring a higher level of meditation are carried out more efficiently at a lower alert level. Also, different sources of pressure during the execution of a task (noise, pressure of time, etc.) require an additional cerebral effort to maintain attention on the task. In contrary on common idea that manual tasks are not require an important cerebral investment, new data in neuroscience show the important implication of the brain in manual tasks [12, 13]. All these facts bring us to the question: to what extent cerebral activity is linked to the muscular activity during the execution of tasks (movements) which may trigger off MSDs while working in a standing position?

The aim of this paper is to present our suggested tool for MSDs prevention. With the collected data from this tool, we wish to identify consistent patterns between brain activities such as suggested in our previous work [14], postures, movements and muscles activity, and then, understand their connection to the development of MSDs. Formatter will need to create these components, incorporating the applicable criteria that follow. Also, this paper shows preliminary results to differentiate inadequate postures in different situations related to the development of MSDs in a context of work in a Flexible Manufacturing System. 


\section{RELATED WORK}

Our tool integrates different sensors in order to characterize a potential progression of MSD. Therefore, this section presents some application of those sensors.

Inertial Measurement Unit (combination of accelerometers, gyroscopes and magnetometers) is widely used in motion measurement and tracking [15], gait analysis [16, 17], inertial navigation and positioning [18-21] as a wearable solution, and even in robotic system using artificial intelligence [22] as an embedded component. Also, this sensor is used to detect human activities (gesture recognition such as user-dependent hand gesture recognition [23] using a dynamic time warping algorithm in order to differentiate each gesture). Then, knowing human gait in the posture analysis is an important parameter. For this reason, we have included an IMU located inside the insole.

This sensor is also used for head motion recognition and tracking for example in vehicle simulator [24]. Sensor fusion is performed by a complementary Kalman filter to weigh the gyroscopic angular rate and gravimetric tilt by accelerometer. This technology is exploited in personal risk assessment in order to evaluate risk of falling [25, 26]. Our tool also integrates IMU in the safety helmet in order to analysis and compute head tilt. Patterns recognition algorithm can then determine the human's intention, and then compute a risk level. This risk level can be evaluated through the analysis of head motion parameters combined to feet center of pressure, and then by differentiation of abnormal posture.

In industrial environment, repeated working activities, noise level, and shift change affect the worker physiological status, his or her state of mind and then possibly result in fatigue, lapses of concentration, vigilance decline, and sleepiness. These abnormal physiological statuses are known as potential threats to the human health and factors of accidents and injuries [27]. For example, Chin-Teng et al. present a real-time wireless EEG-based brain-computer interface system using three EEG electrodes for drowsiness detection in vehicle applications. The reported average of precision and sensitivity achieve $76.9 \%$ and $88.7 \%$, respectively [28]. Our tool uses reusable dry EEG electrodes [29] since they make EEG signal acquisition convenient and easy for real situation. For this reason, our tool includes an EEG, however, this paper doesn't present any result about this device. Finally, our tool could use EMG as to measure muscle activity in the neck-shoulder area as suggested in [8].

The experiments that have been made with the previous tools were made in laboratories within experimental controlled conditions. In our project, we suggest to take our measures in a real context of work. We also suggest a methodology in order to differentiate adequate from inadequate posture which is the main contribution.

\section{SUGGESTED TOOL FOR MSD PREVENTION}

The system, as represented in figure 1, contains: A) an instrumented safety helmet (as presented previously in [14]) which recognizes the head gestures localizes the user during his occupation, and gives information about the brain activity, B) an instrumented insole that gives information about the position and movement of the user's foot, as well as his posture, and C) electromyograms connected to different muscles (neckshoulder area) of the user's body to report his muscles activity, especially those in the neck/shoulder area.

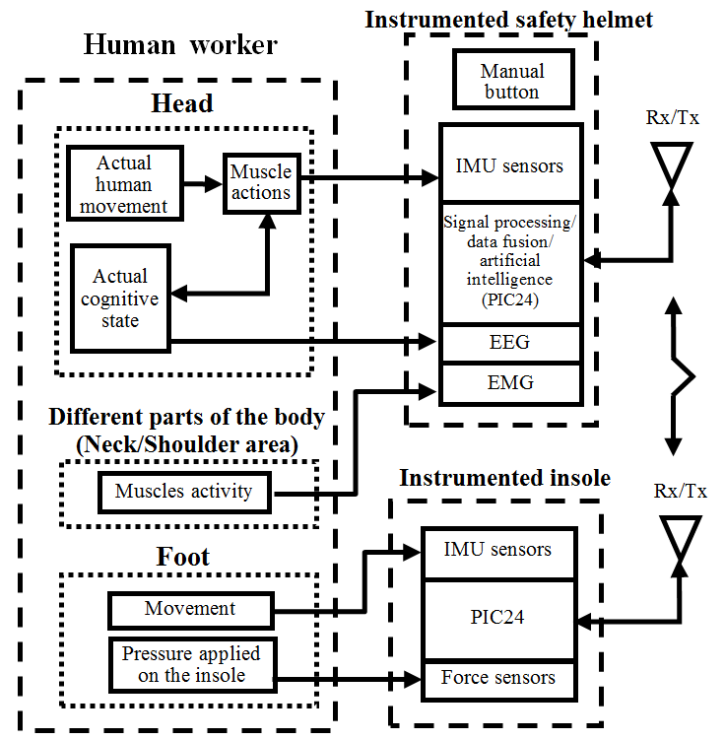

Figure 1: Block diagram of the interactive system

\section{A. The Instrumented Safety Helmet}

The Safety Helmet system, illustrated in Fig. 2, is an inexpensive, non-intrusive, non-invasive, and non-vision-based system. It contains an Inertial Measurement Unit (IMU) that measures the moving object's acceleration, velocity, and orientation, employing a combination of three sensors using three axis measurements: 3DOF accelerometers, 3DOF gyroscopes and 3DOF magnetometers. The accelerometer adopted is the ADXL345. The signals acquired by the IMU are processed by a Kalman filter implemented in a PIC24 microcontroller. The aim is to evaluate human activities recognition especially head movements, translating the movements of the neck/shoulder area. The electronic board is located inside the helmet. It represents the core unit of the artificial intelligence module.

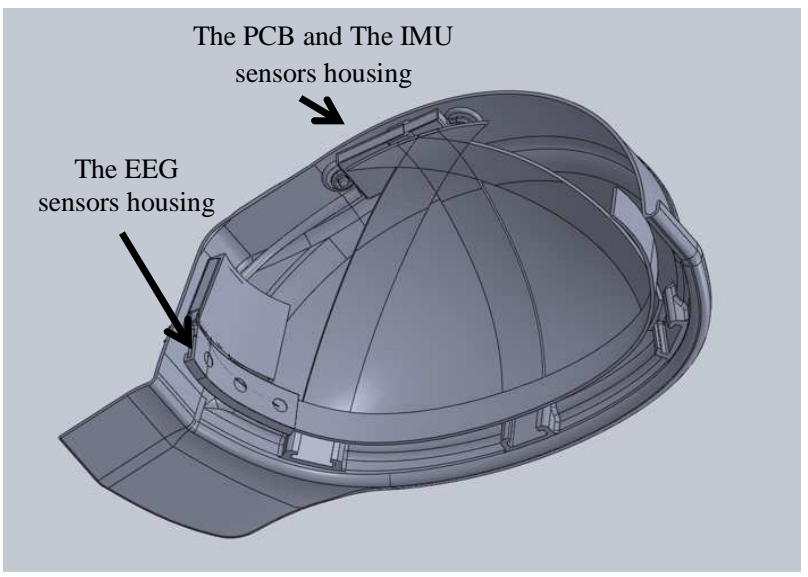

Figure 2: Sectional drawing of the Instrumented Safety Helmet 
Two dry EEG electrodes are inserted on the front of the helmet to capture cerebral physiological signals (in the form of beta waves), to measure the awakening and the concentration of the worker, as well as the eye blink related to fatigue. The EEG electrodes are connected to the microcontroller via an amplifier circuit. Brainwave signals acquired are then processed in order to evaluate the worker's mental state.

\section{B. Neck/shoulder area electromyogram (EMG)}

The EMG signals is amplified $(1000 \times)$, filtered by a bandpass filter $(20-250 \mathrm{~Hz})$, digitized at a sampling rate of $1000 \mathrm{~Hz}$ and recorded with bipolar surface electrodes placed on the bilateral upper-trapeze and anterior deltoid muscles. Before recording EMG, isometric maximal voluntary contractions (MVC) will be perform as a reference for each muscle.

\section{The Instrumented Insole}

The instrumented insole, as shown in Fig. 3, contains four force sensors connected to a PIC24 microcontroller. These sensors help us determining posture of the user. The insole has been placed into a shoe. Besides, an accelerometer was used in order to get information about the foot gestures. It was placed into a housing attached with a clip to the outer side surface of the shoe. The acceleration signal is provided by an ADXL335 connected to a PIC24 microcontroller.

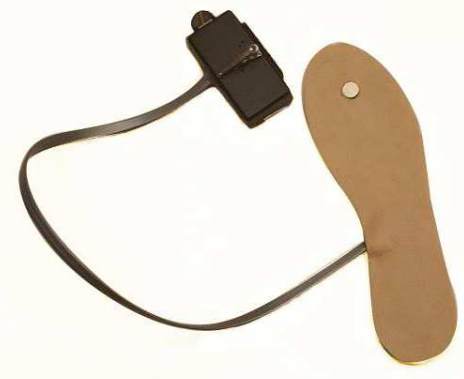

Figure 3: Representation of the Instrumented Insole

\section{DETECTING INADEQUATE POSTURES}

To achieve our objective, we need to take measures of the worker's posture while he's performing his work. Then, we measure $\mathrm{CoP}$ and safety helmet orientation.

\section{A. Measuring Posture from Center of Pressure (CoP)}

The center of pressure $(\mathrm{CoP})$ of the foot is a virtual site of the plantar surface. It represents an average location of all pressures acting on the foot at any given time [30-32]. Several CoP-based measures have been examined in numerous studies $[33,34]$, the aims of these studies can vary from assessing and understanding postural control during, for example, quiet stance or gait, to assessing influences or differences related to age, gender, environmental conditions, health status, etc. [35]. In our case, the aim is to measure the worker's posture. To evaluate this latter we need to calculate the CoP using the data provided by the force sensors located in the insole. One way used to determine the $\mathrm{CoP}$ is by using the following equation [36]:

$$
X_{\mathrm{COP}}=\frac{\sum_{i=1}^{n} X_{i} P_{i}}{\sum_{i=1}^{n} P_{i}} \quad Y_{\mathrm{COP}}=\frac{\sum_{i=1}^{n} Y_{i} P_{i}}{\sum_{i=1}^{n} P_{i}}
$$

where $n$ denotes the total number of sensors, $i$ denotes a certain sensor, $X_{i}, Y_{i}$, are the coordinates of the sensor $i$ on the insole. Our insole includes four FSR sensors (a minimum for computing $\mathrm{CoP})$.

\section{B. Flexible manufacturing system (FMS)}

The workstation is built around a Flexible manufacturing system (FMS), including a Programmable Logic Controller (PLC) and a robot, as shown in Fig. 4. In this workspace, we can find other moving components such as a conveyor, a distributor and a storage system.

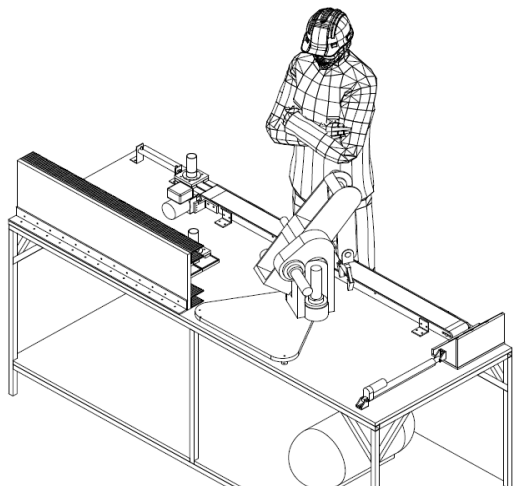

Figure 4: 3D isometric view of the FMS for the hybrid workspace

An automated assembly task is implemented in our FMS: it consists in assembling two different metallic pieces. The assembly task proceeds as following: the distributor pushes the first piece "A" on the conveyor then capacitive and optical sensors will determine the material that composes the piece "A". After this operation, the robot will grab the second piece, "B" on the second distributor, made of the same material than the first one "A". The operator has two main interventions in this whole process: first, filling the distributors with the assembly pieces, and second, manage assembly errors by the recuperation of the pieces. Those interventions could be represented in three main situation shown in Fig. 5.

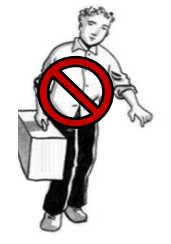

Situation 1
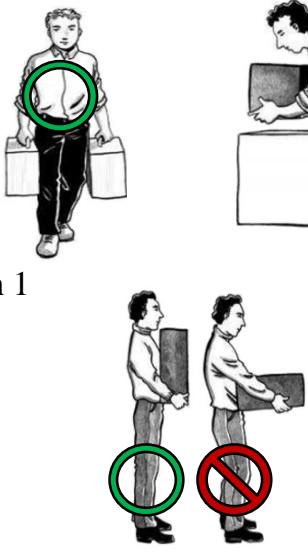

Situation 3

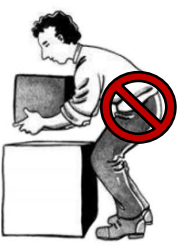

Situation 2

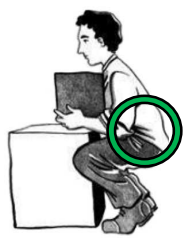

Figure 5: Three situations for evaluating adequate and inadequate posture for handling parts 
In order to evaluate these three situations, the authors test the FMS eight (8) times.

\section{RESULTS}

To make our experiments, we focused on the first task of the operator inside the FMS, which is filling the distributors. We tried to take measures of the operator while he was carrying boxes of assembly pieces: taking them from a conveyor, and putting them on the floor or on the FMS. There is a specific posture that needs to be respected for the work to be done safely as shown in Fig. 5. Indeed, as the boxes containing steal assembly pieces are quite heavy, we need to be even more careful about the posture.
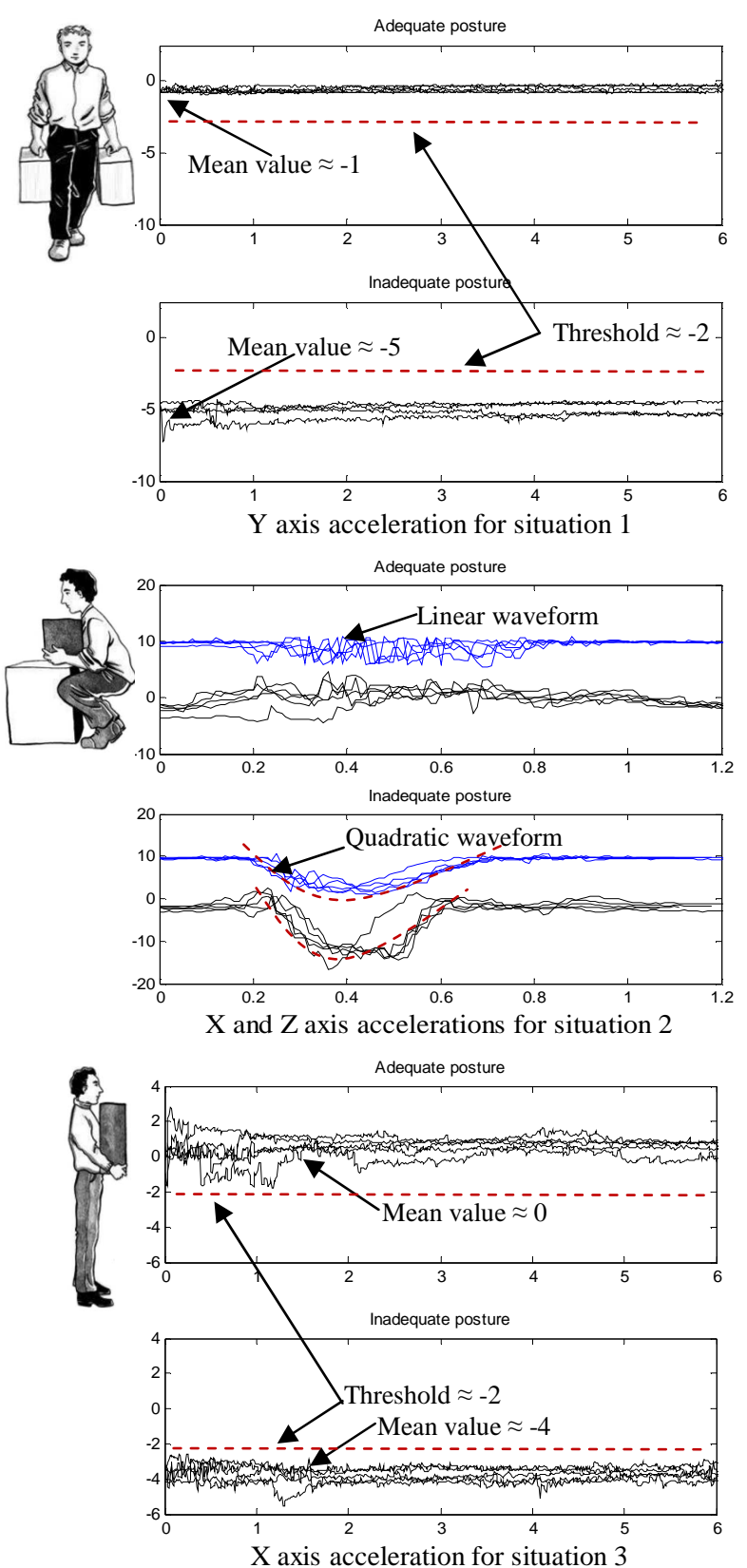

Figure 6: Head orientation for each situation using the instrumented safety helmet
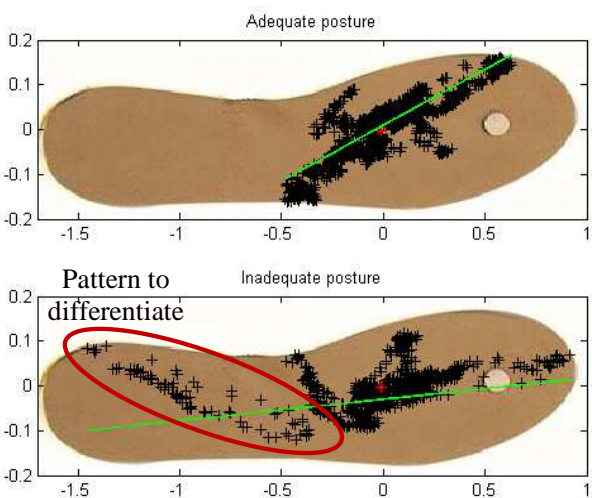

Figure 7: Representation of the CoP with a linear regression and the center of mass (barycenter)

First, in Fig. 6, the acceleration signals (a component of the projection of the gravity vector; the value is depending from the sensor orientation) show a clear difference between each adequate and inadequate posture. A threshold value and a quadratic curve fitting could be used in order to differentiate postures using the instrumented safety helmet. Here, in order to maintain a certain clarity in the figures, only five curves are displayed. Therefore, we need to evaluate the impact of the posture using the instrumented insole.

One way Analysis of Variance (ANOVA) was performed in order to compare the geometry of the $\mathrm{CoP}$ during the execution of the three situations for adequate and inadequate postures. An example of the CoP pattern is given in Fig. 7. On this figure, we can see a linear regression of the CoP with a barycenter. Also, in an inadequate posture, an abnormal pattern could be clearly identified. For this reason, an ANOVA analysis was performed using other statistic data such as kurtosis, variance and RMS value in order to differentiate the abnormal pattern.

The ANOVAs result is reported as an F-statistic with its associated degrees of freedom and its p-value. The null hypothesis $H_{0}$ is that all the means of the CoP distribution (Fig. 7 ) is similar. Given that the null hypothesis is rejected if $F>$ $F_{\text {critical }}$ at the 0.05 level of significance, the computed test statistic $F$ in our work is:

$\mathrm{F}=4.5 \quad$ (variance in $\mathrm{X}$ axis in 1 ), $p=0.049<0.05$,

$\mathrm{F}=8.95$ (kurtosis in $\mathrm{Y}$ axis in 2), $p=0.0086<0.05$ and

$\mathrm{F}=9.14$ (kurtosis in $\mathrm{Y}$ axis in 3), $p=0.0128<0.05$,

which is more than $F_{\text {critical }}$; for situation 1,2 and 3 respectively. This analysis of variance, given in Fig. 8, leads to the conclusion that there has a significant effect related to the inadequate posture. Those results combined with Fig. 6 could increase algorithm robustness.

\section{CONCLUSION}

In this paper, we suggest a new tool for evaluating MSDs development. We specifically present results from both the instrumented insole and safety helmet to assess inadequate posture which can lead to MSD. This tool gives us the head position and the CoP under the foot. The three situations described in this paper could be differentiate using these data. In future works, the ANOVA analysis will be used for the design of a pattern recognition algorithm implemented in our medical electronic record. 

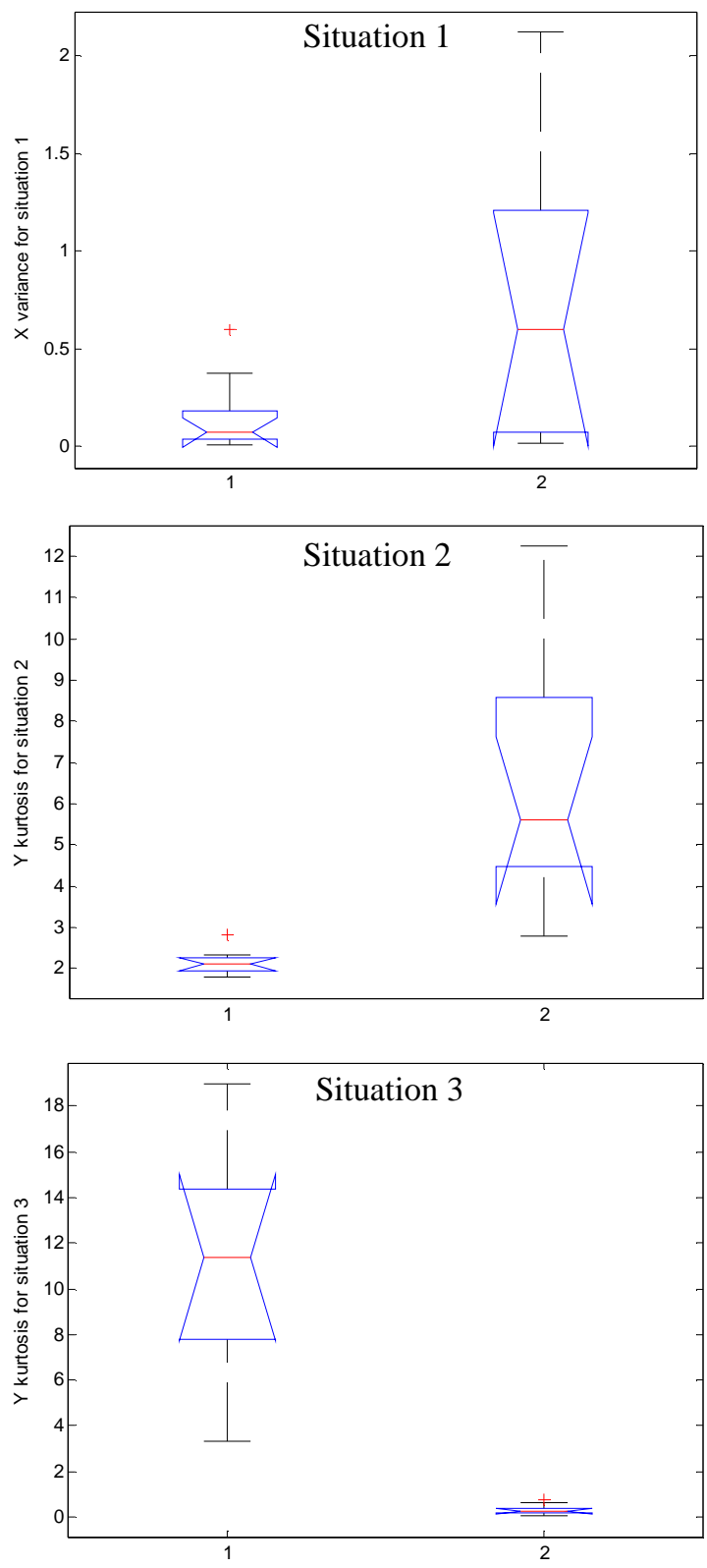

Figure 8: ANOVA for all situations

\section{REFERENCES}

[1] P. Côté, G. van der Velde, J. D. Cassidy, L. J. Carroll, S. Hogg-Johnson, L. W. Holm, et al., "The Burden and Determinants of Neck Pain in Workers: Results of the Bone and Joint Decade 2000 - 2010 Task Force on Neck Pain and Its Associated Disorders," Journal of Manipulative and Physiological Therapeutics, vol. 32, pp. S70-S86, 2009.

[2] M. N. Baliki, T. J. Schnitzer, W. R. Bauer, and A. V. Apkarian, "Brain Morphological Signatures for Chronic Pain," PLoS ONE, vol. 6, p. e26010, 2011.

[3] S. Ngomo, G. Leonard, and C. Mercier, "Influence of the amount of use on hand motor cortex representation: effects of immobilization and motor training," Neuroscience, vol. 220, pp. 208-14, 2012.

[4] S. Ngomo, C. Mercier, L. J. Bouyer, A. Savoie, and J.-S. Roy, "Alterations in central motor representation increase over time in individuals with rotator cuff tendinopathy," Clinical Neurophysiology, vol. 126, pp. 365-371, 2// 2015.

[5] P. Ranasinghe, Y. Perera, D. Lamabadusuriya, S. Kulatunga, N. Jayawardana, S. Rajapakse, et al., "Work related complaints of neck, shoulder and arm among computer office workers: a cross-sectional evaluation of prevalence and risk factors in a developing country," Environmental Health, vol. 10, p. 70, 2011.

[6] D. M. Antle and J. N. Côté, "Relationships between lower limb and trunk discomfort and vascular, muscular and kinetic outcomes during stationary standing work," Gait \& Posture, vol. 37, pp. 615-619, 4// 2013.

[7] T. R. Waters and R. B. Dick, "Evidence of Health Risks Associated with Prolonged Standing at Work and Intervention Effectiveness," Rehabilitation Nursing, pp. n/an/a, 2014.

[8] D. E. Gregory and J. P. Callaghan, "Prolonged standing as a precursor for the development of low back discomfort: An investigation of possible mechanisms," Gait \& Posture, vol. 28, pp. 86-92, 2008.

[9] D. Lafond, A. Champagne, M. Descarreaux, J.-D. Dubois, J. M. Prado, and M. Duarte, "Postural control during prolonged standing in persons with chronic low back pain," Gait \& Posture, vol. 29, pp. 421-427, 4// 2009.

[10] R. W. Aas, H. Tuntland, A. HolteKari, C. Røe, T. Lund, S. Marklund, et al., "Workplace interventions for neck pain in workers," Cochrane Database of Systematic Reviews, vol. 13, p. CD008160, 2011.

[11] B. Nasseroleslami, H. Lakany, and B. A. Conway, "EEG signatures of arm isometric exertions in preparation, planning and execution," NeuroImage, vol. 90, pp. 1-14, 4/15/ 2014.

[12] H. Sun, T. M. Blakely, F. Darvas, J. D. Wander, L. A. Johnson, D. K. Su, et al., "Sequential activation of premotor, primary somatosensory and primary motor areas in humans during cued finger movements," Clinical Neurophysiology.

[13] B. Berger, T. Minarik, G. Liuzzi, F. C. Hummel, and P. Sauseng, "EEG Oscillatory Phase-Dependent Markers of Corticospinal Excitability in the Resting Brain," BioMed Research International, vol. 2014, p. 8, 2014.

[14] P. Li, R. Meziane, M. J.-D Otis, H. Ezzaidi, and P. Cardou, "A Smart Safety Helmet using IMU and EEG sensors for worker fatigue detection," presented at the IEEE International Symposium on RObotic and SEnsors Environments (ROSE), Timisoara - Romania, 2014.

[15] A. Godfrey, R. Conway, D. Meagher, and O. L. G, "Direct measurement of human movement by accelerometry," Med Eng Phys, vol. 30, pp. 1364-86, Dec 2008.

[16] Y. Ohtaki, K. Sagawa, and H. Inooka, "A method for gait analysis in a daily living environment by body-mounted 
instruments," JSME International Journal Series C, vol. 44, pp. 1125-1132, 2001.

[17] S. J. M. Bamberg, A. Y. Benbasat, D. M. Scarborough, D. E. Krebs, and J. A. Paradiso, "Gait analysis using a shoe-integrated wireless sensor system," IEEE Transactions on Information Technology in Biomedicine, vol. 12, pp. 413-423, 2008.

[18] F. Zampella, M. Khider, P. Robertson, and A. Jiménez, "Unscented Kalman filter and Magnetic Angular Rate Update (MARU) for an improved Pedestrian DeadReckoning," presented at the IEEE/ION Position, Location and Navigation Symposium, Myrtle Beach, SC, 2012.

[19] T. Gadeke, J. Schmid, M. Zahnlecker, W. Stork, and K. D. Muller-Glaser, "Smartphone pedestrian navigation by foot-IMU sensor fusion," presented at the Ubiquitous Positioning, Indoor Navigation, and Location Based Service, 2012.

[20] M. Angermann, P. Robertson, T. Kemptner, and M. Khider, "A high precision reference data set for pedestrian navigation using foot-mounted inertial sensors," presented at the International Conference on Indoor Positioning and Indoor Navigation (IPIN), Zürich, Switzerland, 2010.

[21] S. Wan and E. Foxlin, "Improved pedestrian navigation based on drift-reduced MEMS IMU chip," presented at the Proceedings of the 2010 International Technical Meeting of The Institute of Navigation, San Diego, CA, 2010.

[22] C. Smith and H. I. Christensen, "Wiimote robot control using human motion models," presented at the IEEE/RSJ International Conference on Intelligent Robots and Systems, 2009.

[23] J. Liu, L. Zhong, J. Wickramasuriya, and V. Vasudevan, "uWave: Accelerometer-based personalized gesture recognition and its applications," Pervasive and Mobile Computing, vol. 5, pp. 657-675, 2009.

[24] E. M. Foxlin, "Head tracking relative to a moving vehicle or simulator platform using differential inertial sensors," presented at the AeroSense, 2000.

[25] B. Najafi, K. Aminian, F. Loew, Y. Blanc, and P. A. Robert, "Measurement of stand-sit and sit-stand transitions using a miniature gyroscope and its application in fall risk evaluation in the elderly," Biomedical Engineering, IEEE Transactions on, vol. 49, pp. 843-851, 2002.

[26] M. J.-D. Otis and B.-A. J. Menelas, "Toward an augmented shoe for preventing falls related to physical conditions of the soil," presented at the IEEE International Conference on Systems, Man, and Cybernetics (SMC), South Korea, Seoul, 2012.

[27] J. Samra, M. Gilbert, M. Shain, and D. Bilsker. (2012, March 14). Thirteen psychosocial risk (PSR) factors. Available:

http://www.guardingmindsatwork.ca/info/risk_factors

[28] L. Chin-Teng, C. Che-Jui, L. Bor-Shyh, H. ShaoHang, C. Chih-Feng, and I. J. Wang, "A Real-Time Wireless Brain-Computer Interface System for Drowsiness Detection," IEEE Transactions on Biomedical Circuits and Systems, vol. 4, pp. 214-222, 2010.

[29] K. Lee and S. Yang, "Method and apparatus for quantitatively evaluating mental states based on brain wave signal processing system," US20080177197 A1, 2008.

[30] D. JA., "Gait analysis in the science of rehabilitation. Washington, D.C.: Dept. of Veterans Affairs;", 1998.

[31] P. R. Gill-Body KM, Parker SW, Krebs DE, "Rehabilitation of balance in two patients with cerebellar dysfunction," Physical Therapy, vol. 77, pp. 534-52, 1997.

[32] T. o. H. Lin Shu, Yangyong Wa ng, Qiao Li, Dav id Dagan Feng, Fellow, IEEE, and Xiaoming Tao, Member, IEEE, "In-Shoe Plantar Pressure Measurement a nd Analysis System Based on Fabric Pressure Sensing Array," IEEE Transactions on Information Technology in Biomedicine, vol. 14, 2010.

[33] A. W. Eytan M. Debbi , Yulia Goryachev, Ziva Yizhar, Elhanan Luger, Ronen Debi , Amir Haim "In-shoe center of pressure: Indirect force plate vs. direct insole measurement," 2012.

[34] S. K. M. A. Nussbaum, "Evaluation of Two Approaches for Aligning Data Obtained from a Motion Capture System and an In-Shoe Pressure Measurement System," 2014.

[35] B.-J. M. Hsue, F.; Su, F.-C., "The dynamic balance of the children with cerebral palsy and typical developing during gait. Part I: Spatial relationship between COM and COP trajectories," Gait Posture, vol. 29, pp. 465-470, 2009.

[36] A. F. Ruhe, R.; Walker, B, "The test-retest reliability of centre of pressure measures in bipedal static task conditions-A systematic review of the literature," Gait Posture, vol. 32, pp. 436-445, 2010. 